\title{
ON A CONTRACTIVITY CONDITION IN FIXED POINT THEORY AND THE THEORY OF SELECTIONS
}

\author{
PAVEL V. SEMENOV \\ Moscow City Pedagogical University, Math. Dept. \\ 2-nd Selskochozjastvenny pr. 4, Moscow 129226, Russia \\ E-mail:pavels@orc.ru
}

1. Introduction. A common point in two theories, fixed point theory and the theory of selections, is the consecutive procedure of constructing the resulting answer. In both cases one first tries to find an approximative answer, i.e., an $\varepsilon$-fixed point or an $\varepsilon$-selection. The next step deals with an improvement of the accuracy $\varepsilon$. And the final step usually relates to passing to the limit as $\varepsilon \rightarrow 0^{+}$.

In the present note, special attention is paid to a purely analytic expression for the consecutive improvement procedure (see Section 2, below). The applications to selection theory for non-convex-valued mappings, to fixed point theory and to minimax theory, are presented in Sections 3, 4, and 5, respectively.

The geometric progression with functional quotient $\alpha:(0, \infty) \rightarrow(0, \infty)$ is defined by setting

$$
\alpha_{0}(t)=t, \quad \alpha_{1}(t)=\alpha(t) \cdot t, \quad \alpha_{n+1}(t)=\alpha\left(\alpha_{n}(t)\right) \cdot \alpha_{n}(t) \quad \text { for } n \in \mathbb{N} .
$$

For a constant mapping $\alpha(\cdot) \equiv q$, we obtain the usual geometric progression.

Definition 1. A mapping $\alpha:(0, \infty) \rightarrow(0,1)$ is said to be $G$-summable (or geometrically summable) at a point $t>0$ if the series $\sum_{n=0}^{\infty} \alpha_{n}(t)$ is convergent.

So, the key question in the present note concerns analytical conditions which guarantee the $G$-summability of the quotient $\alpha:(0, \infty) \rightarrow(0,1)$ in a neighborhood of zero. Clearly, if the function $\alpha_{1}(t)=\alpha(t) \cdot t$ is decreasing in some neighborhood of zero, then it suffices to verify the $G$-summability of $\alpha:(0, \infty) \rightarrow(0,1)$ at a single point $t>0$.

The work was partially supported by RFBR grant No 05-01-00993

The paper is in final form and no version of it will be published elsewhere. 
2. Let us consider the following constraints on $\alpha:(0, \infty) \rightarrow(0,1)$ introduced by Reich [9]:

$$
\begin{aligned}
& \forall t>0 \quad \limsup _{s \rightarrow t^{+}} \alpha(s)<1, \\
& \forall t \geq 0 \quad \limsup _{s \rightarrow t^{+}} \alpha(s)<1 .
\end{aligned}
$$

Lemma 2. Property $\left(\mathrm{R}^{\sharp}\right)$ implies G-summability.

Proof. From $\alpha_{n+1}(t)=\alpha\left(\alpha_{n}(t)\right) \cdot \alpha_{n}(t)$ and $\alpha:(0, \infty) \rightarrow(0,1)$ we see that for any fixed positive $t$ the decreasing sequence $\left\{\alpha_{n}(t)\right\}_{n=1}^{\infty}$ tends to some $l \geq 0$. Passing to the limit in $\alpha_{n+1}(t)=\alpha\left(\alpha_{n}(t)\right) \cdot \alpha_{n}(t)$ one can check that $l=0$, thanks to the property $(\mathrm{R})$. Then the property $\left(\mathrm{R}^{\sharp}\right)$ implies that $\alpha\left(\alpha_{n}(t)\right) \leq q$ for some $0<q<1$ and for all sufficiently large $n$. Hence $\sum_{n=N}^{\infty} \alpha_{n}(t) \leq \alpha_{N}(t) \cdot \sum_{k=0}^{\infty} q^{k}<\infty$.

In view of Lemma 2 , in what follows we consider the functions $\alpha:(0, \infty) \rightarrow(0,1)$ possessing property $(\mathrm{R})$, but with $\lim _{s \rightarrow 0^{+}} \alpha(s)=1$. In other words, it can be assumed that $\alpha(0)=1$.

LEMma 3. If $\alpha^{\prime}(0)$ exists and differs from $-\infty$, then $\alpha(\cdot)$ is not $G$-summable in any neighborhood of zero.

Proof. Let $\alpha(t)>1-C t$ for some $C>0$ and for all sufficiently small $t>0$. We take a $t<2 C^{-1}$ and write $\alpha_{i}(t)=\alpha_{i}$. Then

$$
\frac{1}{\alpha_{i+1}}-\frac{1}{\alpha_{i}}=\frac{\alpha_{i}-\alpha_{i+1}}{\alpha_{i} \cdot \alpha_{i+1}}=\frac{1-\alpha\left(\alpha_{i}\right)}{\alpha_{i+1}}<\frac{C \alpha_{i}}{\alpha_{i+1}}<\frac{C}{1-C \alpha_{i}}<2 C
$$

since $\alpha\left(\alpha_{i}\right)>1-C \alpha_{i}$ and $\alpha_{i+1}>\alpha_{i}\left(1-C \alpha_{i}\right)$.

Hence,

$$
\frac{1}{\alpha_{n}}=\left(\frac{1}{\alpha_{n}}-\frac{1}{\alpha_{n-1}}\right)+\left(\frac{1}{\alpha_{n-1}}-\frac{1}{\alpha_{n-2}}\right)+\ldots+\left(\frac{1}{\alpha_{1}}-\frac{1}{\alpha_{0}}\right)+\frac{1}{\alpha_{0}}<A n+B
$$

and $\alpha_{n}>\frac{1}{A n+B}$ for some constants $A$ and $B$.

Below, it will be convenient to introduce the function $\phi(t)=1-\alpha(t)$ and its inverse function $\psi=\phi^{-1}$. Clearly, here we assume that $\alpha(\cdot)$ is decreasing in some neighborhood of zero. The proof of the lemma below is a direct generalization of the proof of Lemma 3 .

LEMMA 4. Let

$$
\lim \inf _{s \rightarrow 0^{+}}\left\{\frac{1}{\phi(t \cdot(1-\phi(t))}-\frac{1}{\phi(t)}: 0<t<s\right\}>\lambda>0 .
$$

Then $\alpha_{n}(t)<\psi\left(\frac{1}{\lambda n+\mu(t)}\right)$ for all sufficiently small $t>0$ and for some constants $\mu=\mu(t)$. Sketch of proof. As in Lemma 3 let $\alpha_{i}(t)=\alpha_{i}$. Then $\alpha_{i+1}=\alpha\left(\alpha_{i}\right) \cdot \alpha_{i}=\alpha_{i}\left(1-\phi\left(\alpha_{i}\right)\right)$ and

$$
\frac{1}{\phi\left(\alpha_{i+1}\right)}-\frac{1}{\phi\left(\alpha_{i}\right)}=\frac{1}{\phi\left(\alpha_{i} \cdot\left(1-\phi\left(\alpha_{i}\right)\right)\right)}-\frac{1}{\phi\left(\alpha_{i}\right)}>\lambda
$$

for all sufficiently small $t>0$. Hence $\frac{1}{\phi\left(\alpha_{n}\right)}>\lambda n+\mu$, or $\alpha_{n}<\psi\left(\frac{1}{\lambda n+\mu}\right)$.

Applying the Lagrange theorem one can easily show that the hypothesis of Lemma 4 holds for functions with $\liminf _{t \rightarrow 0^{+}} \frac{t \phi^{\prime}(t)}{\phi(t)}>0$ and with decreasing derivative $\phi^{\prime}$. In fact, 
for some $c_{t} \in(t-t \phi(t), t)$ we have

$$
\frac{1}{\phi(t \cdot(1-\phi(t))}-\frac{1}{\phi(t)}=\frac{\phi(t)-\phi(t-t \phi(t))}{\phi(t) \cdot \phi(t-t \phi(t))}=\frac{t \phi(t) \phi^{\prime}\left(c_{t}\right)}{\phi(t) \cdot \phi(t-t \phi(t))}>\frac{t \phi^{\prime}(t)}{\phi(t)}
$$

because $\phi(t-t \phi(t))<\phi(t)$ and $\phi^{\prime}\left(c_{t}\right)>\phi^{\prime}(t)$.

Note that such restrictions trivially hold for $\phi(t)=C t^{p}, 0<p<1$. The estimate for the rate of convergence of iterations of such functions is a well known fact. It appeared in several articles $[2,5,17]$. Here we present the theorem for iterations of functions without any power-law majorants.

THEOREM 5. Let $f:(0, \infty) \rightarrow(0, \infty)$ be an arbitrary function with the following properties as $\tau \rightarrow \infty$ :

$$
\begin{gathered}
f(\infty)=\infty \\
f(\tau)=o(\tau), \\
-1<f^{\prime}(\tau) \leq \text { const. } \\
\int_{\tau}^{\infty} \frac{d s}{e^{f(s)}}<\infty .
\end{gathered}
$$

Let

$$
\psi(t)=t^{1+f(-\ln t) /(-\ln t)}
$$

for all sufficiently small $t>0$. Then the function $\psi(\cdot)$ is invertible in a neighborhood of zero, the function $\alpha=1-\psi^{-1}$ admits no power-law majorants of the type $1-C t^{p}$, $p \in(0,1)$, and the function $\alpha=1-\psi^{-1}$ is geometrically summable.

Proof.

$$
\psi(t)=e^{\ln t(1+f(-\ln t) /(-\ln t))}=e^{-g(\tau)}, \quad \tau=-\ln t, \quad g(\tau)=\tau+f(\tau) .
$$

Assumption (3) implies that the function $g(\cdot)$ is increasing at infinity, and, hence, it has an inverse function, say $h(\cdot)$. Then, for $\phi=\psi^{-1}$, we have $\phi(t)=e^{-h(\tau)}, t=-\ln t$. Indeed,

$$
\phi(\psi(t))=e^{-h(-\ln (\psi(t)))}=e^{-h(g(\tau))}=e^{\ln t}=t .
$$

If, to the contrary, the inequality $\alpha(t)=1-\phi(t)<1-C t^{p}, 0<p<1$, holds in a neighborhood of zero, then $\phi(t)>C t^{p}$, and $\psi(t)<C_{1} t^{1 / p}$. Thus, in view of (5) and (2) we see that

$$
1 \leftarrow \frac{\psi(t)}{t}<C_{1} t^{1 / p-1} \rightarrow 0, \quad t \rightarrow 0,
$$

which is a contradiction.

Now, let us verify the $G$-summability of $\alpha=1-\psi^{-1}$. Since the derivative of $g(\cdot)$ admits a finite and positive majorant, the derivative of the inverse function $h(\cdot)$ has a positive infimum at infinity. Since $\phi(t)=e^{-h(\tau)}, \tau=-\ln t$, we see that $\frac{t \phi^{\prime}(t)}{\phi(t)}=t \cdot(-h(-\ln t))^{\prime}=$ $h^{\prime}(\tau) \geq \lambda>0$.

So, Lemma 4 gives the upper estimates $\alpha_{n}(t)<\psi\left(\frac{1}{\lambda n+\mu(t)}\right)$. The series $\sum_{n=0}^{\infty} \psi\left(\frac{1}{\lambda n+\mu(t)}\right)$ is convergent or divergent simultaneously with the series $\sum_{n=0}^{\infty} \psi\left(\frac{1}{n}\right)$ by the comparison 
test and the fact that $\frac{\psi(t)}{t} \rightarrow 1$ as $t \rightarrow 0$. Remembering that $\tau=-\ln t=-\ln \left(n^{-1}\right)=$ $\ln n$, and in view of (5), we see that $\psi\left(\frac{1}{n}\right)=\frac{1}{n^{1+f(\tau) / \tau}}$. Therefore $\sum_{n=0}^{\infty} \psi\left(\frac{1}{n}\right)<\infty$ iff $\int \frac{d x}{x^{1+f(\ln x) / \ln x}}<\infty$ by the integral test for convergence.

After the substitution $z=\ln x$, we obtain the integral $\int \frac{d z}{e^{f(z)}}$, which is finite precisely by assumption (4).

The set of all functions with properties (1)-(4) in Theorem 5 forms an infinitedimensional convex subset, which includes such examples as $f(\tau)=C \ln \tau, C>1$, and $f(\tau)=C \tau^{p}(\ln \tau)^{q}, 0<p<1, q \geq 0, C>0$. A direct calculation of the functions $\psi, \phi$, and $\alpha$ is a non-trivial problem. It is rather simple for $f(\tau)=2 C \sqrt{\tau}$. In this case, $\alpha(t)=1-e^{-\left(\sqrt{C^{2}-\ln t}-C\right)^{2}}$.

3. For a nonempty closed subset $P \subset Y$ of a Banach space $(Y,\|\cdot\|)$ and for an open ball $D \subset Y$ of radius $r$ with a nonempty intersection $P \cap D$, we define

$$
\delta(P, D)=\sup \{\operatorname{dist}(q, P) / r: q \in \operatorname{conv}(P \cap D)\} .
$$

Clearly, for a convex set $P$ the equality $\delta(P, D)=0$ holds for each open ball $D$ with $P \cap D \neq \emptyset$. Thus, one can define the value of the function of nonconvexity $\alpha_{P}$ for a set $P$ at a number $r>0$ as $\alpha_{P}(r)=\sup \{\delta(P, D)\}$, where the supremum is taken over the set of all open balls $D$ of radius $r$ with a nonempty intersection $P \cap D$. For a closed set $P$, its convexity is equivalent to the identity $\alpha_{P}(\cdot) \equiv 0$. The more $\alpha_{P}$ differs from zero, the "less convex" the set $P$ is. Graphs of Lipschitz functions or, graphs of polynomials with suitable constraints for their coefficients are rather typical examples of sets with $G$-summable functions of nonconvexity [14]. Note that in general $0 \leq \alpha_{P}(r) \leq 2$ and the deep results of $\mathrm{V}$. Klee imply that $Y$ is an inner product space iff $0 \leq \alpha_{P}(\cdot) \leq 1$ for each closed subset $P$ of $Y[7]$.

Recall that lower semicontinuity of a multi-valued mapping $F: X \rightarrow Y$ between topological spaces $X$ and $Y$ means that for each $x \in X$ and $y \in F(x)$, and each open neighborhood $U(y)$, there exists an open neighborhood $V(x)$ such that $F\left(x^{\prime}\right) \cap U(y) \neq \emptyset$, whenever $x^{\prime} \in V(x)$. Applying the Axiom of Choice to the family of nonempty intersections $F\left(x^{\prime}\right) \cap U(y), x^{\prime} \in V(x)$, we see that LSC mappings are exactly those which admit local (noncontinuous) selections. A single-valued mapping $f: X \rightarrow Y$ is said to be a selection of a multi-valued mapping $F: X \rightarrow Y$ if $f(x) \in F(x), x \in X$. For a metric range space $Y$ and for $\varepsilon>0$ a single-valued mapping $f: X \rightarrow Y$ is said to be an $\varepsilon$-selection of $F: X \rightarrow Y$ if $\operatorname{dist}(f(x), F(x))<\varepsilon, x \in X$. Note that for the class of upper semicontinuous mappings between metric spaces the term " $\varepsilon$-selection" is frequently used for mappings $f: X \rightarrow Y$ whose graphs are $\varepsilon$-close to the graph of $F$ [11].

TheORem 6. Let $F: X \rightarrow Y$ be a multi-valued lower semicontinuous mapping from a paracompact space $X$ into a Banach space $Y$. Let $\alpha:(0, \infty) \rightarrow(0,1)$ be a common majorant for all the functions of nonconvexity $\alpha_{F(x)}, x \in X$. Let $f: X \rightarrow Y$ be a single-valued continuous $\varepsilon$-selection of $F$. Then there exists a single-valued continuous $\alpha(\varepsilon) \varepsilon$-selection $g$ of $F$.

Sketch of proof. For each $x \in X$, the open ball $D(f(x) ; \varepsilon)$ intersects the set $F(x)$. The 
continuity of $f$ and the lower semicontinuity of $F$ together imply the lower semicontinuity of the mappings

$x \mapsto F(x) \cap D(f(x) ; \varepsilon) ; \quad x \mapsto \operatorname{conv}(F(x) \cap D(f(x) ; \varepsilon)) ; \quad x \mapsto \overline{\operatorname{conv}}(F(x) \cap D(f(x) ; \varepsilon))$.

Applying the classical Michael selection theorem to the last multi-valued mapping we find a single-valued continuous $g: X \rightarrow Y$ such that $g(x) \in \overline{\operatorname{conv}}(F(x) \cap D(f(x) ; \varepsilon)), x \in X$. By the definition of the function of nonconvexity $\alpha_{F(x)}$ we see that $\operatorname{dist}(g(x) ; F(x)) \leq$ $\alpha_{F(x)}(\varepsilon) \varepsilon<\alpha(\varepsilon) \varepsilon$, i.e., $g$ is an $a(\varepsilon) \varepsilon$-selection of $F$.

The direct repeated application of Theorem 6 together with the results of Section 2 gives a selection theorem for non-convex-valued mappings.

Theorem 7 ([14]). Let $F: X \rightarrow Y$ be a lower semicontinuous mapping from a paracompact space $X$ into a Banach space $Y$. Let $\alpha:(0, \infty) \rightarrow(0,1)$ and $\beta:(0, \infty) \rightarrow(0,1)$ be two functions such that $\alpha$ is geometrically summable and $\alpha_{F(x)}(t)<\beta(t)<\alpha(t), x \in X$, $t>0$. Then $F$ admits a continuous single-valued selection.

Note that, for a constant $\alpha(\cdot) \equiv q$, Theorem 7 was proved by Michael [7].

4. Let $k:(0, \infty) \rightarrow(0,1)$. A multi-valued mapping $F$ associating to each point $x$ of a metric space $(X, d)$ some nonempty subset $F(x) \subset X$, is called a $k$-contraction if $H(F(x), F(y)) \leq k(d(x, y)) \cdot d(x, y), x \neq y$. This inequality means that, for each $\varepsilon>$ $k(d(x, y)) \cdot d(x, y)$, the sets $F(x)$ and $F(y)$ lie in the $\varepsilon$-neighborhood of each other.

Reich $[9,10]$ showed that property $(\mathrm{R})$ guarantees the existence of a fixed point for an arbitrary compact-valued $k$-contraction $F$ of a complete metric space. He also stated a (still) open problem concerning the possibility of removing the compactness condition, $[10,12]$. In $[1,8]$, the positive answer was given when $\left(\mathrm{R}^{\sharp}\right)$ is substituted for $(\mathrm{R})$. Repeating the well-known approximation procedure for fixed points, [3], and applying the results of Section 2 we obtain

THEOREM $8([15])$. Let $k(\cdot)$ be a function possessing the property $(R)$ and admitting a geometrically summable majorant. Then each closed-valued $k$-contraction $F$ of a complete metric space $(X, d)$ has a fixed point.

As to fixed point theorems for self-mappings of topologically "nice" domains instead of contractions, the following Glicksberg type theorem is true.

THeOREM 9 ([14]). Let $K$ be an ANR compact subset of a Banach space and let $K$ have the fixed point property with respect to single-valued continuous mappings. Then $K$ has the fixed point property with respect to the upper semicontinuous multi-valued mappings whose values have geometrically summable functions of nonconvexity.

5. The John von Neumann-Ky Fan-Sion minimax theorem [16] stated the equality $\max _{x \in X} \min _{y \in Y} f(x, y)=\min _{y \in Y} \max _{x \in X} f(x, y)$ for convex subcompacta $X$ and $Y$ of locally convex topological linear spaces and for a real-valued function $f: X \times Y \rightarrow \mathbb{R}$ which satisfies the following assumptions:

(a) $\forall c \in \mathbb{R} \forall x_{0} \in X$ the set $\left\{y \in Y: f\left(x_{0}, y\right) \leq c\right\}$ is convex and compact;

(b) $\forall d \in \mathbb{R} \forall y_{0} \in Y$ the set $\left\{x \in X: f\left(x, y_{0}\right) \geq d\right\}$ is convex and compact. 
There exist many generalizations of this fundamental theorem. Most of them deal with various kinds of generalized, topological, or axiomatically defined convexities in (a) and (b). The key role in all approaches to minimax theorems is played by the socalled Knaster-Kuratowski-Mazurkiewicz (KKM) principle. Having in mind this principle, many authors exploited not precisely the convexity assumption, but only the basic hereditary property that the intersection of convex sets is also convex $[4,6,18]$.

For the sets $P$ with a fixed majorant of their functions of nonconvexity an analog of another principal property, somewhat symmetrical to intersections holds. Namely, that the unions of directly ordered family of arbitrary convex sets are also convex sets.

Lemma 10 ([13]). Let $P_{1} \subset P_{2} \subset \ldots \subset P_{n} \subset \ldots$ be a sequence of subsets in a Banach space $B$ such that $\alpha_{P_{n}}(t)<\beta(t)<\alpha(t), n \in \mathbb{N}, t>0$. Then the function of nonconvexity $\alpha_{P}$ of the closure $P=\operatorname{Cl}\left(\bigcup_{n} P_{n}\right)$ of their union is pointwise less than $\alpha$.

In [13], instead of versions of the KKM principle, the selection theory (see Section 3) was used as a base for obtaining minimax theorems. Such minimax theorems include the cases in which the finite intersections of sublevel and suplevel sets are nonempty, but non-connected.

Theorem 11 ([13]). Let $f: X \times Y \rightarrow \mathbb{R}$ be a function on the Cartesian product of two AR-subcompacta in Banach spaces $B_{1}$ and $B_{2}$. Let $\alpha:(0, \infty) \rightarrow(0,1)$ be a geometrically summable function. Assume that:

$\left(\mathrm{a}^{\prime}\right) \forall c \in \mathbb{R} \forall x_{0} \in X$ the function of nonconvexity for the set $\left\{y \in Y: f\left(x_{0}, y\right) \leq c\right\}$ is pointwise less than $\alpha$;

$\left(\mathrm{b}^{\prime}\right) \forall d \in \mathbb{R} \forall y_{0} \in Y$, the function of nonconvexity of the set $\left\{x \in X: f\left(x, y_{0}\right) \geq d\right\}$ is pointwise less than $\alpha$.

Then $\max _{X}\left(\min _{Y} f(x, y)\right)=\min _{Y}\left(\max _{X} f(x, y)\right)$.

The author would like to express his gratitude to prof. J. Jachymski for helpful discussions and to the referee for her/his very useful comments and remarks.

\section{References}

[1] P. Z. Daffer, H. Kaneko, Fixed points of generalized contractive multi-valued mappings, J. Math. Anal. Appl. 192 (1995), 655-666.

[2] P. Z. Daffer, H. Kaneko, W. Li, On a conjecture of S. Reich, Proc. Amer. Math. Soc. 124 (1996), 3159-3162.

[3] J. Dugundji, A. Granas, Fixed Point Theory, Vol. I, Monografie Matematyczne 61, PWN, Warszawa 1982.

[4] J. Kindler, Topological intersection theorems, Proc. Amer. Math. Soc. 117 (1993), 1003-1011.

[5] G. Koenigs, Recherches sur les integrals de certaines équations fonctionnelles, Ann. Sci. École Norm. Sup. 3 (1884), 3-41.

[6] M. Lassonde, On the use of KKM multifunctions in fixed point theory and related topics, J. Math. Anal. Appl. 97 (1983), 151-201.

[7] E. Michael, Paraconvex sets, Math. Scand. 7 (1959), 372-376. 
[8] N. Mizoguchi, W. Takahashi, Fixed point theorems for multi-valued mappings on complete metric spaces, J. Math. Anal. Appl. 141 (1989), 177-188.

[9] S. Reich, Fixed points of contractive functions, Boll. Un. Mat. Ital. (4) 5 (1972), 26-42.

[10] S. Reich, Some fixed point problems, Atti Accad. Naz. Lincei Rend. Cl. Sci. Fis. Mat. Natur. (8) 57 (1974), 194-198.

[11] S. Reich, Approximate selections, best approximations, fixed points, and invariant sets, J. Math. Anal. Appl. 62 (1978), 104-113.

[12] S. Reich, Some problems and results in fixed point theory, in: Topological Methods in Nonlinear Functional Analysis (Toronto 1982), Contemp. Math. 21, Amer. Math. Soc., Providence 1983, 179-187.

[13] D. Repovš, P. V. Semenov, A minimax theorem for functions with possibly nonconnected intersections of sublevel sets, J. Math. Anal. Appl. 314 (2006), 537-545.

[14] P. V. Semenov, Fixed-points theorems under controllable rejection of the convexity of values of a multi-valued mapping, Mat. Sb. 189:3 (1998), 141-160 (in Russian); English transl.: Sb. Math. 189 (1998), 461-480.

[15] P. V. Semenov, On fixed points of multi-valued contractions, Funktsional. Anal. i Prilozhen. 36:2 (2002), 89-92 (in Russian); English transl.: Funct. Anal. Appl. 36 (2002), 159-161.

[16] M. Sion, On general minimax theorem, Pacific J. Math. 8 (1958), 171-176.

[17] W. J. Thron, Sequences generated by iterations, Trans. Amer. Math. Soc. 96 (1960), 38-53.

[18] Wu Wen-Tsun, A remark on the fundamental theorem in the theory of games, Sci. Record (N.S.) 3 (1959), 229-233. 
